\title{
Somatotypes trajectories during adulthood and their association with COPD phenotypes
}

\author{
Miguel J. Divo (1) ${ }^{1}$, Marta Marin Oto ${ }^{2}$, Ciro Casanova Macario ${ }^{3}$, Carlos Cabrera \\ Lopez $^{4}$, Juan P. de-Torres ${ }^{2}$, Jose Maria Marin Trigo $\mathbb{1}^{5,6}$, Craig P. Hersh ${ }^{1,7}$, \\ Ana Ezponda Casajús ${ }^{8}$, Cherie Maguire ${ }^{7}$, Victor M. Pinto-Plata \\ Francesca Polverino ${ }^{10}$, James C. Ross ${ }^{1}$, Dawn DeMeo ${ }^{1,7}$, Gorka Bastarrika ${ }^{8}$, \\ Edwin K. Silverman ${ }^{1,7}$ and Bartolome R. Celli (10)
}

\section{ABSTRACT}

Rationale: Chronic obstructive pulmonary disease (COPD) comprises distinct phenotypes, all characterised by airflow limitation.

Objectives: We hypothesised that somatotype changes - as a surrogate of adiposity - from early adulthood follow different trajectories to reach distinct phenotypes.

Methods: Using the validated Stunkard's Pictogram, 356 COPD patients chose the somatotype that best reflects their current body build and those at ages 18, 30, 40 and 50 years. An unbiased group-based trajectory modelling was used to determine somatotype trajectories. We then compared the current COPD-related clinical and phenotypic characteristics of subjects belonging to each trajectory.

Measurements and main results: At 18 years of age, $88 \%$ of the participants described having a lean or medium somatotype (estimated body mass index (BMI) between 19 and $23 \mathrm{~kg} \cdot \mathrm{m}^{-2}$ ) while the other $12 \%$ a heavier somatotype (estimated BMI between 25 and $27 \mathrm{~kg} \cdot \mathrm{m}^{-2}$ ). From age 18 onwards, five distinct trajectories were observed. Four of them demonstrating a continuous increase in adiposity throughout adulthood with the exception of one, where the initial increase was followed by loss of adiposity after age 40. Patients with this trajectory were primarily females with low BMI and $D_{\text {LCO }}$ (diffusing capacity of the lung for carbon monoxide). A persistently lean trajectory was seen in $14 \%$ of the cohort. This group had significantly lower forced expiratory volume in $1 \mathrm{~s}\left(\mathrm{FEV}_{1}\right), D_{\mathrm{LCO}}$, more emphysema and a worse BODE (BMI, airflow obstruction, dyspnoea and exercise capacity) score thus resembling the multiple organ loss of tissue (MOLT) phenotype.

Conclusions: COPD patients have distinct somatotype trajectories throughout adulthood. Those with the MOLT phenotype maintain a lean trajectory throughout life. Smoking subjects with this lean phenotype in early adulthood deserve particular attention as they seem to develop more severe COPD.

@ERSpublications

In smoking-related COPD, somatotype trajectories are associated with the final COPD phenotype. Specifically, the "pink puffer" or multiorgan loss of tissue (MOLT) phenotype occurs where the patient remains lean. https://bit.ly/2YNhwfu

Cite this article as: Divo MJ, Marin Oto M, Casanova Macario C, et al. Somatotypes trajectories during adulthood and their association with COPD phenotypes. ERJ Open Res 2020; 6: 00122-2020 [https://doi.org/10.1183/23120541.00122-2020].

This article has supplementary material available from openres.ersjournals.com.

Received: 18 March 2020 | Accepted after revision: 15 June 2020

Copyright $\odot$ ERS 2020. This article is open access and distributed under the terms of the Creative Commons Attribution Non-Commercial Licence 4.0. 


\section{Introduction}

Chronic obstructive pulmonary disease (COPD) is recognised as a phenotypically heterogeneous clinical syndrome characterised by chronic respiratory symptoms, different degrees of structural pulmonary abnormalities, lung function impairment and extrapulmonary manifestations. COPD is usually diagnosed after the 5th decade of life, although its origin begins in the early stages of life as a result of the cumulative effect of different exposures and complex interactions between genetic, epigenetic and age-related factors in susceptible individuals [1]. This long latency period from the initial exposures to clinical diagnosis makes it difficult to study the natural history of COPD. In recent years we have learned that the development of airway obstruction can follow different trajectories starting at an early age [2]; however, little is known as to why individuals develop distinct COPD phenotypes.

The initial description of distinct COPD phenotypes dates back to the 1950s with the characterisation of the predominantly emphysematous phenotype commonly called the "pink puffer" and a predominantly bronchitic phenotype called the "blue bloater" [3]. More recently, studies using hypothesis-free cluster analysis resulted in the description of three to five discrete COPD phenotypes [4-8]. While the reproducibility of specific clusters among different COPD cohorts is modest, the classic "pink puffer" and "blue bloater" phenotypes tend to recur across multiple cohorts [9]. More recently, the original "pink puffer" phenotype was refined and the term multiorgan loss of tissue phenotype (MOLT) was coined [10]. These patients are characterised by having more emphysema, worse airflow obstruction and higher BODE (BMI, airflow obstruction, dyspnoea and exercise capacity) score, being more prone to exacerbations, suffering a higher mortality and having a lower body mass index (BMI).

Interestingly, in all of these studies one of the most salient clinical traits that differentiates between these phenotypes is the BMI, suggesting that adipose tissue may have a modulating effect in response to cigarette smoking [11]. In support of this idea, investigators from two landmark epidemiological cohorts (The Nurses' Health Study and the Health Professionals Follow-up Study) demonstrated that adiposity changes throughout the life course predict the subsequent risk for chronic diseases and mortality [12-14]. They employed a Group-Based Multi-trajectory Modelling technique to participants' recalled body builds at different age points to identify distinct subgroups of participants with a similar trajectory of body shape from childhood to age 50 .

We hypothesised that changes in somatotypes - as a surrogate of the degree of adiposity - throughout adult life follow different trajectories to reach the subsequent COPD phenotype. More specifically, we sought to investigate if patients with the MOLT phenotype were signalled from an early age to manifest a different somatic response to the effects of cigarette smoke.

\section{Methods}

Study population

Participants were recruited from the BODE collaborative group (Tenerife, Gran Canarias, Pamplona and Zaragoza sites) and from the COPDGene study (Brigham and Women's Hospital - Boston clinical site). In the BODE cohort, regular follow-up examination visits occur at $\sim 12$ - to 24-month intervals. Subjects in COPDGene-Boston cohort were included for this study at the follow-up visit, $\sim 5$ years after the initial enrolment. In both cohorts, COPD was defined by a history of smoking of at least 10 pack-years and a ratio of forced expiratory volume in $1 \mathrm{~s}\left(\mathrm{FEV}_{1}\right)$ to forced vital capacity (FVC) of $<0.7$ measured $20 \mathrm{~min}$ after the administration of albuterol. Details of the inclusion and exclusion criteria for both cohorts have been described elsewhere $[15,16]$. For the present analyses, participants were also asked to complete the validated Stunkard's somatotype questionnaire [17] (figure 1) during their visit that occurred between October 2013 and June 2017. The ethics committee at each of the participating centres approved the study, and all patients provided written informed consent before enrolment.

Affiliations: 'Pulmonary and Critical Care Division, Brigham and Women's Hospital, Harvard Medical School, Boston, Massachusetts, USA. ${ }^{2}$ Pulmonary Dept, Clinica Universidad de Navarra, Pamplona, Spain. ${ }^{3}$ Pulmonary Dept and Research Unit, Hospital Universitario La Candelaria, Universidad de La Laguna, Santa Cruz de Tenerife, Spain. ${ }^{4}$ Respiratory Service, Hospital Universitario de Gran Canaria Dr. Negrin, Canary Islands, Spain. ${ }^{5}$ Respiratory Service, Hospital Universitario Miguel Servet, Zaragoza, Spain. ${ }^{6} \mathrm{CIBER}$ Enfermedades Respiratorias, Instituto Investigación Sanitaria, Madrid, Spain. ${ }^{7}$ Channing Division of Network Medicine, Boston, MA, USA. ${ }^{8}$ Dept of Radiology, Clinica Universidad de Navarra, Pamplona, Spain. ${ }^{9}$ Pulmonary and Critical Care Division, Baystate Medical Center, Springfield, MA, USA. ${ }^{10}$ Asthma and Airway Disease Research Center, University of Arizona, Tucson, NM, USA.

Correspondence: Miguel J. Divo, 75 Francis Street, Pulmonary and Critical Care Division, Brigham and Women's Hospital and Spaulding Rehabilitation Hospital, Boston, MA 02115, USA. E-mail: mdivolabwh. harvard.edu 
Which diagram below best depicts your outline at a given age?

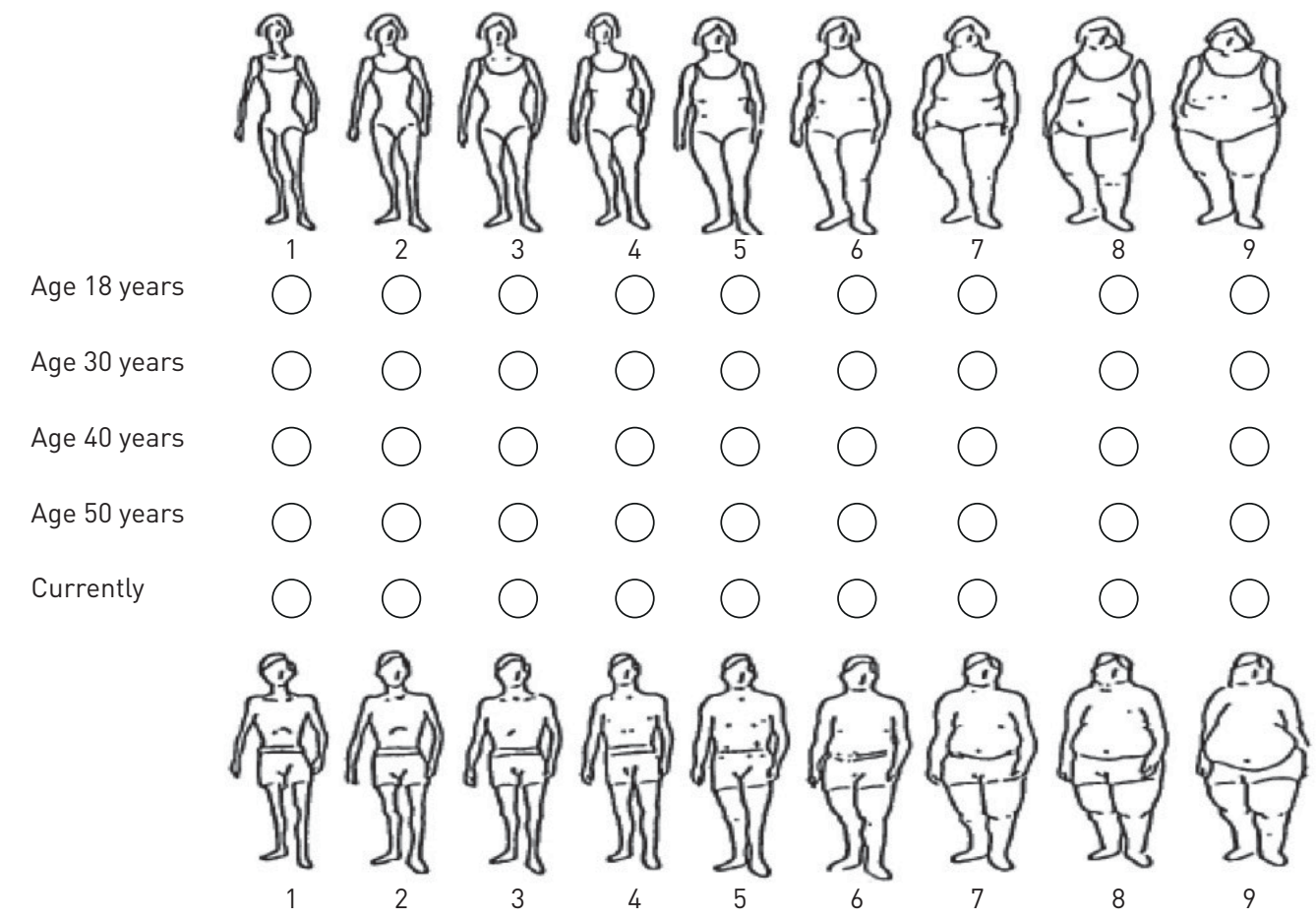

FIGURE 1 Stunkard's Pictogram. Figure drawings used to assess body shape at ages 18, 30, 40 and 50, and at study evaluation.

\section{Assessment of body shape}

The Stunkard's Pictogram is a validated instrument of nine line drawings of human figures reflecting the spectrum of body physiques or somatotypes (figure 1) [17]. Participants were asked to select the somatotype diagram that best depicted their current body build and those at ages 18, 30, 40 and 50 years. The validity of long-term recall of somatotypes and their correlation with related BMI was assessed among 181 participants aged 71-76 years in the Third Harvard Growth Study [18]. In this study they reported a correlation of 0.63 for men and 0.74 for women between the chosen figure and the subject's recall of height and weight $>50$ years ago. The utility of this instrument was also validated in large epidemiology studies to estimate adiposity trajectories across the life course $[12-14,19,20]$.

\section{Ascertainment of outcomes and other covariates}

Demographics, smoking history (age of smoking initiation, number of cigarettes per day, date of smoking cessation), anthropometrics (assessed by the BMI in $\mathrm{kg} \cdot \mathrm{m}^{-2}$ ), pulmonary function tests $\left(\mathrm{FEV}_{1} / \mathrm{FVC} \mathrm{FEV}_{1} \%\right.$ predicted and diffusing capacity of the lung for carbon monoxide $\left(D_{\mathrm{LCO}}\right) \%$ predicted $)$ and 6-min walking distance tests were performed according to international guidelines [21,22]. The BODE index was calculated as previously reported [16]. Emphysema was assessed by visual quantification of lung parenchyma from available chest computed tomography studies by two independent expert radiologists (GB and AE) using validated criteria [23, 24]. The extent of emphysema was graded from 0 to 4 , with a grade of 0 indicating no emphysema, grade 1 indicating $1-25 \%$, grade 2 indicating 25-50\%, grade 3 indicating $50-75 \%$ and grade 4 indicating the presence in $>75 \%$ of emphysema in the lungs.

\section{Statistical analysis}

For categorical variables reported as proportions we used the Chi-squared test. For continuous variables reported as means (95\% CI) we used the t-test. To present a more meaningful interpretation of the Stunkard's Pictogram scale to BMI in $\mathrm{kg} \cdot \mathrm{m}^{-2}$, we fitted a linear regression model with current BMI as the outcome, somatotype at the time of study visit as predictor and sex as covariate and interaction term. We then used the regression coefficient and the reported somatotype at the time of visit to convert the nine-points scale and reported the strength of correlation between the pictogram and BMI for each sex.

We used a group-based trajectory modelling approach to identify subjects that share similar somatotype trajectories using subjects' chosen somatotype scores at the time of visit and those at ages 18, 30, 40 and 
50. Thus, subjects are assigned to the trajectory group to which they have the highest probability of belonging. Based on the trajectory modelling analysis and clinical interpretability, we selected the model with five trajectories for this analysis (see details in the supplementary material). After group trajectories were assigned, we then compared the phenotypic characteristics (sex, BMI, FEV $\%$ pred, $\mathrm{FEV}_{1} / \mathrm{FVC}$,

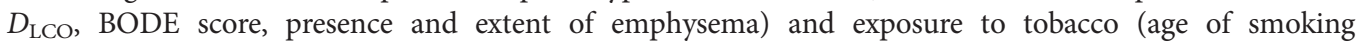
initiation, pack-years) between each of the five trajectory-based groups to determine if somatotype trajectories are associated with specific COPD phenotypes. Differences in these phenotypic characteristics amongst the five trajectory-based groups were tested using ANOVA for continuous variables and a Chi-squared test for categorical variables. Missing data were imputed using the low-rank matrix approximation provided as the Automated Data Imputation function in JMP.

We compared subjects' characteristics from the BODE and COPDGene cohorts to prevent selection bias and enhance internal validity. Statistical significance was considered at the level of $\mathrm{p}<0.05$. For the group-based trajectory modelling analysis we used the Stata (StataCorp. 2017, Stata Statistical Software: Release 15; StataCorp LLC, College Station, TX, USA) plug-in command "traj" available at www.andrew. cmu.edu/user/bjones/ [25]. All other analyses were performed using SAS JMP Pro ${ }^{\circledR}$ software, version 14.0 (SAS Institute, Cary, NC, USA).

\section{Results}

\section{Descriptive}

The combined cohorts included 356 subjects with COPD with a mean age of 67 years (95\% CI 66-68) and mean BMI of $27.2 \mathrm{~kg} \cdot \mathrm{m}^{-2}$ (95\% CI 26.7-27.7); $68 \%$ were males. According to the Global Initiative for Chronic Obstructive Lung Disease (GOLD) spirometric classification, 33\% had mild, 45\% had moderate and $22 \%$ had severe or very severe airflow limitation.

\section{Pictogram correlation}

The Pearson correlation between the current body build and the BMI at time of visit was 0.77 (95\% CI 0.73-0.81). The prediction equation for BMI obtained from fitting the current somatotype score is:

$$
\text { BMI=17.6 (95\% CI 16.7-18.5) + } 1.9(95 \% \text { CI 1.8-2.1) } \times \text { somatotype score }
$$

This regression formula suggests that every increase in somatotype unit represents an increase of almost $2 \mathrm{~kg} \cdot \mathrm{m}^{-2}$ in BMI (figure 2 and supplementary figure E1). There was no statistical difference in the parameter estimates between males and females.
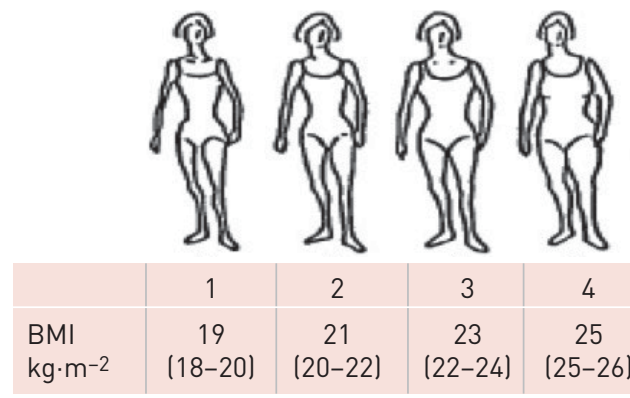

3
23
$(22-24)$

4
25
$25-26$
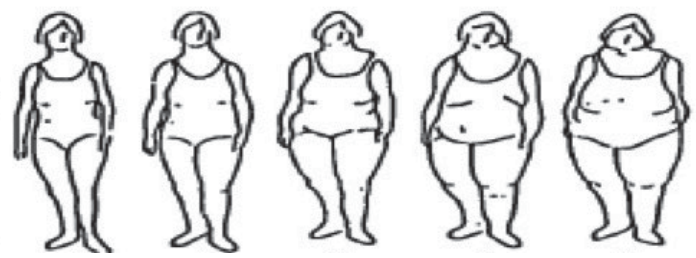

\begin{tabular}{c|c}
5 & 6 \\
\hline 27 & 29 \\
$(27-28)$ & $(29-30)$
\end{tabular}

7
31
$(30-32)$

8
34
$(32-35)$

9
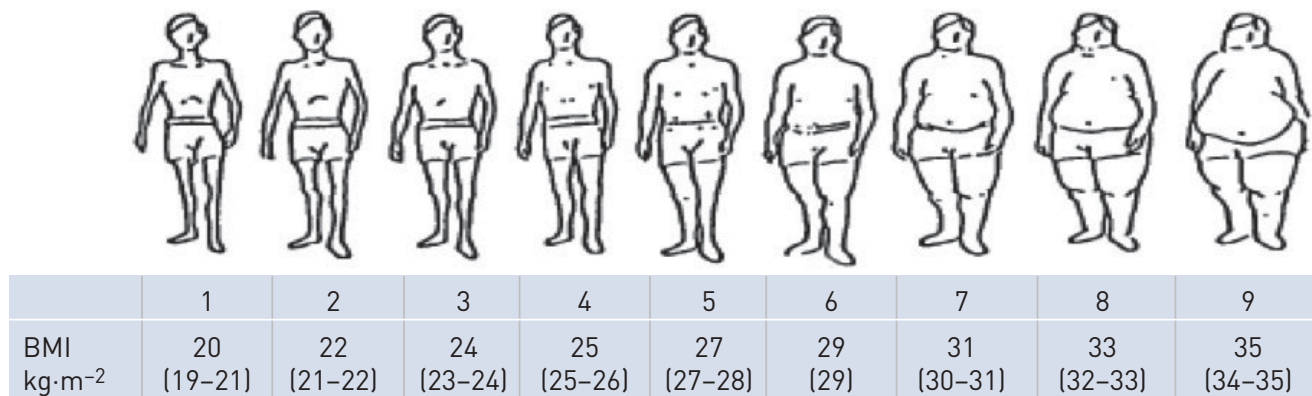

1
20
$19-21)$

22

22

3
24
$(23-24$

4
25
$(25-26)$

5
27
$(27-28)$

6
29
$129]$

7
31
$(30-31)$

8
33
$(32-33)$

9
35
$(34-35)$

FIGURE 2 Predicted BMI for each somatotype's values. We assigned a body mass index (BMI) value to each somatotype based on the regression formula obtained from participants' current BMI and somatotype score. Values are expressed as mean and $95 \%$ confidence interval. 


\section{Body shapes trajectories}

As shown in figure 3, we named each trajectory group using a descriptor based on their initial point at age 18 (intercept) and the directionality of the trajectory as Lean-Flat, Lean-Increase, Medium-Increase and Medium-Parabolic and Heavy-Increase. The first group with a "Lean-Flat" trajectory comprises 14\% $(n=49)$ of the cohort, the second group with a "Lean-Increase" trajectory comprises $21 \%(n=74)$, the third group or "Medium-Increase" trajectory has $38 \% \quad(n=138)$ subjects, the fourth group or "Medium-Parabolic" trajectory has $15 \%(n=54)$ subjects and the final group with a "Heavy-Increase" trajectory has 41 subjects or $12 \%$ of the cohort.

From table 1, we can observe that subjects in all five groups have similar mean age and similar proportions of current smokers, they initiated their smoking habit at a similar age and have the same cumulative smoking history. The majority have a tendency towards gaining and then sustaining adiposity, although at a different rate and starting point, during adult life except for the "Lean-Flat" and Medium-Parabolic" groups. Phenotypically the three progressive adiposity groups share similar characteristics, namely less obstruction, higher BMI and $D_{\text {LCO }}$ and lower BODE scores. In contrast, the Lean-Flat group is distinctively different in the trajectory and phenotypic characteristics compared to the other groups. They remain lean throughout adult life and are significantly more obstructed, have a lower

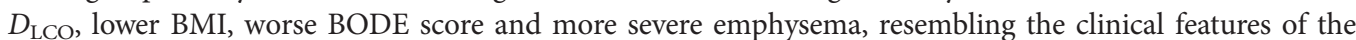
implosive or MOLT phenotype described by CeLLi et al. [10]. Next to the Lean-Flat group is the Medium-Parabolic trajectory group, sharing some of the characteristics of the former including a significantly lower BMI and second lowest $D_{\mathrm{LCO}}$. However, this group has a higher proportion of females (49\%) and a particular trajectory demonstrating an initial increase in adiposity followed by loss after age 40 .

Supplementary table E2 shows that the 301 patients from the BODE cohort and 55 from the COPDGene-Boston cohort were similar in their clinical characteristics except that subjects from the BODE cohort were slightly younger.
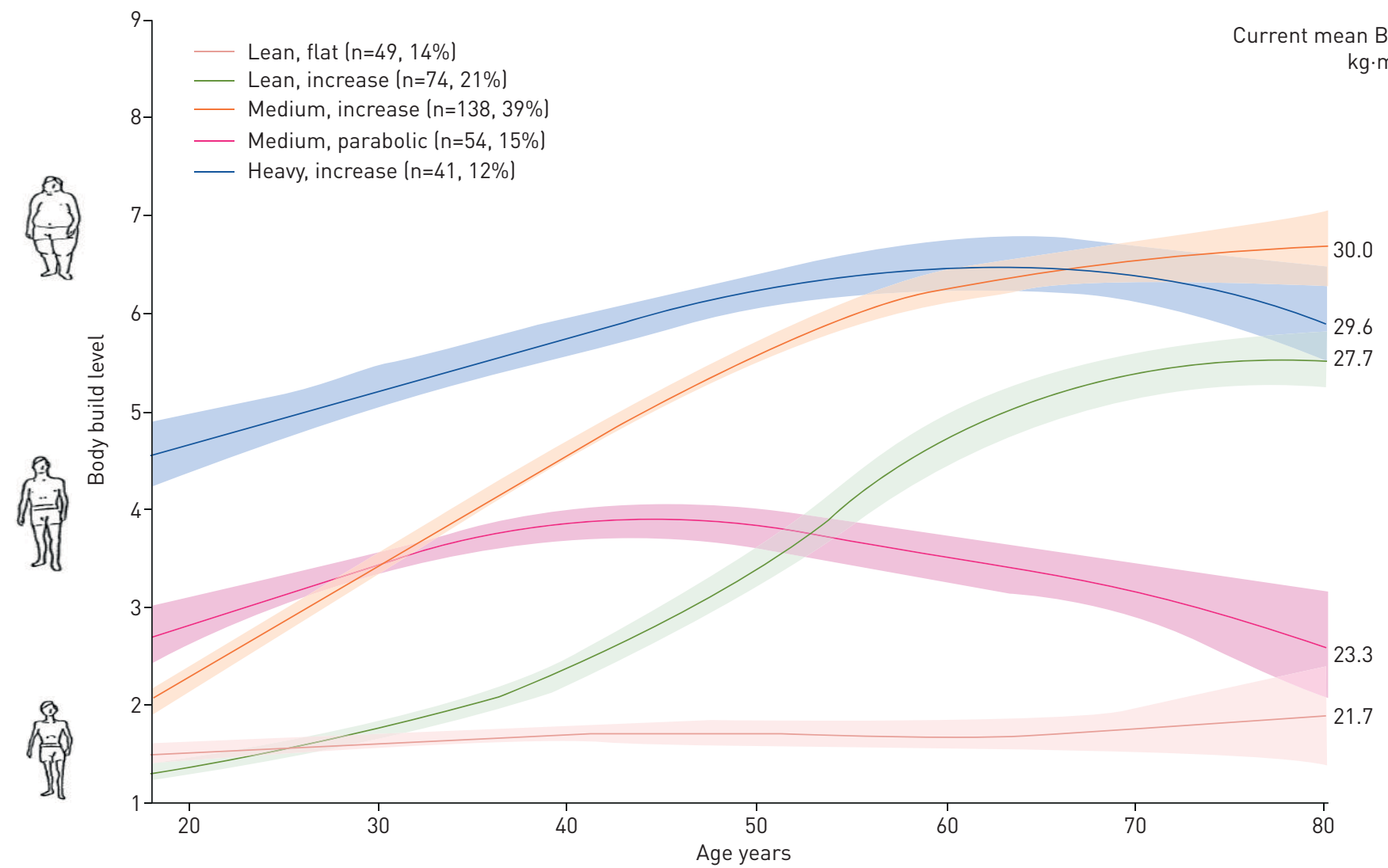

FIGURE 3 Somatotype trajectories throughout adult life. Each line represents a trajectory estimate and the shaded bands the $95 \%$ confidence interval fit of the mean. 
TABLE 1 Comparison of phenotypic features for each of the five trajectory-based groups

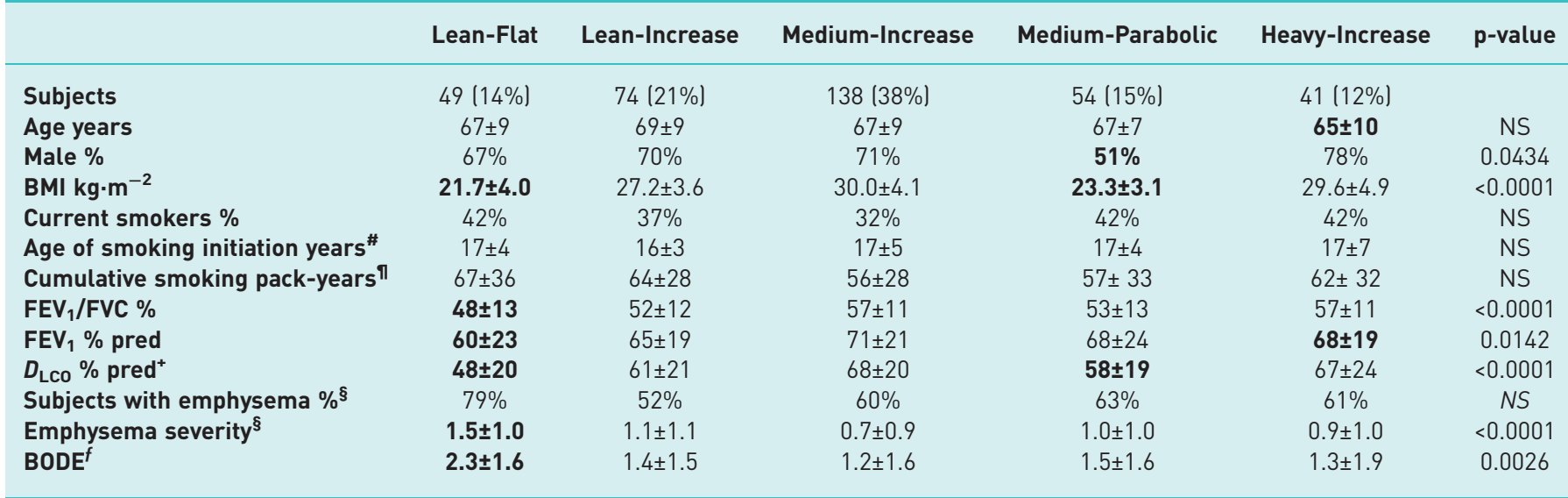

Data are presented as mean $\pm \mathrm{SD}$, unless otherwise stated. Group comparison was performed using ANOVA for continuous variables, a Chi-squared test for categorical variables. Those values that conferred a significant difference are highlighted in bold. Results in the table reflect estimates of parameters with imputed missing values. BMI: body mass index; FEV ${ }_{1}$ : forced expiratory volume in 1 s; FVC: forced vital capacity; $\%$ pred: $\%$ predicted; $D_{\mathrm{LCO}}$ : diffusing capacity of the lung for carbon monoxide; BODE: BMI, airflow obstruction, dyspnoea and exercise capacity. \#: Data missing in 25 subjects with chronic obstructive pulmonary disease (COPD); " : data missing in 75 subjects: 5 (10\%) in Lean-Flat, 20 (27\%) in Lean-Increase, 24 (17\%) in Medium-Increase, 13 (24\%) in Medium-Parabolic, 11 (27\%) in Heavy-Increase group; ${ }^{+}$: data missing in 71 subjects: 7 (17\%) in Lean-Flat group, $16(22 \%)$ in Lean-Increase, 30 (23\%) in Medium-Increase, 14 (26\%) in Medium-Parabolic, 4 $(10 \%)$ in Heavy-Increase group; $\S$ : data missing in 113 subjects: 21 (43\%) in Lean-Flat, 25 (34\%) in Lean-Increase, 41 (30\%) in Medium-Increase, $13(24 \%)$ in Medium-Parabolic, $13(32 \%)$ in Heavy-Increase group; ${ }^{f}$ : data missing in 11 subjects.

\section{Discussion}

Our study identified five distinct somatotype trajectories throughout adulthood in subjects with smoking-related COPD. Importantly, those trajectories related to the final phenotypic expression of the disease.

In our cohort, $88 \%$ of participants started with a lean body shape (estimated BMI between 20 and $24 \mathrm{~kg} \cdot \mathrm{m}^{-2}$ ) at age 18. But after the $3 \mathrm{rd}$ and 4 th decade of life, $59 \%$ reported a steady increase in somatotype, a trend that is not unique to patients with COPD $[13,26]$. What is novel in this study is that patients with the MOLT phenotype in late adulthood were found to have followed a lean (lack of adiposity) trajectory throughout life, starting from an early age. The $14 \%$ of participants who demonstrated this trajectory have many of the features of the MOLT phenotype, namely a lower BMI, worse airway obstruction, lower $D_{\mathrm{LCO}}$, more severe emphysema and a worse BODE score than subjects in the other groups (table 1).

Another noteworthy finding is the parabolic trajectory observed in $15 \%$ of the cohort, where there is an initial adiposity gain that peaked at age 40 , followed by progressive loss thereafter. This subgroup had the largest proportion of females (49\%) and also manifest some of the features of the MOLT phenotype, in this case the second lowest $\mathrm{BMI}$ and $D_{\mathrm{LCO}}$ (figure 3 and table 1). Interestingly, this trajectory was also observed in the Nurses' Health Study [13], with women showing a steeper decline in adiposity after age 40. The investigators in that study named the trajectory as 'Lean-Stable', but little attention or explanation was given to its meaning. We speculate that since it is observed primarily in women, it may relate to life events after age 40 , such as the post-childbearing stage. It is unlikely to be related to cigarette smoking, as smoking was not more prevalent in the 11000 participants with this trajectory.

The association between subjects' BMI and COPD has been extensively studied. First, in two longitudinal studies of asymptomatic young and middle-aged adults, a low BMI at baseline was associated with a higher risk for developing airway obstruction during the 10 [27] and 15 [25] years of follow-up. Second, there is strong evidence that a low BMI is associated with increased mortality risk from respiratory causes in COPD patients $[28,29]$ as well as in the general population [30]. Third, the prevalence of certain comorbidities differs significantly between COPD patients with low and high BMI [31], and in addition the BMI is a salient feature varying between phenotypes in cluster analysis studies [4-7]. However, this evidence cannot explain what separates COPD patients into different phenotypes. Based on our findings and suggestions from previous reviews $[11,32,33]$ we can speculate that some of the differences in COPD phenotypes may relate to the accumulation (or lack thereof) of adipose tissue and its response to the repetitive and cumulative effect of cigarette smoking. It is well known that adipose tissue is not just an 
inert store of energy, but rather another organ capable of modulating inflammation via signalling molecules (adipokines) and also a source of mesenchymal stem cells that can participate in tissue repair $[11,34,35]$. It is also known that the fat mass and obesity-associated (FTO) genotype influences early adulthood and midlife weight [36-38] and in COPD is associated with low body mass and low lung function [39].

This study has several limitations. Our design, referred to as retrolective [40], where the outcome variables to define the specific phenotypes were measured during the study visit and the exposure (somatotypes at different age points) was collected by recall but treated as repeated measures over time, raises the possibility of recall and survivor bias. To reduce recall bias, we decided to use Stunkard's Pictogram. This instrument was validated by demonstrating a strong correlation of the long-term recall (up to 50 years) of subjects' somatotype with their historical BMI values [18]. Further, seminal epidemiological studies have demonstrated the validity of this simple instrument to draw somatotype trajectories [12-14, 38]. To enhance recall precision, we chose specific age points to draw the trajectories. We started at age 18, because it is the age when maximal height has already been reached and therefore changes in somatotype would likely represent changes in weight. After age 18, the scale defined each decade of life. It is also possible that we are showing the trajectories of COPD survivors, as our cohort has only $3 \%$ of subjects under the age of 50, and individuals with severe airway obstruction were likely underrepresented in this cohort. Thus, our findings reflect the trajectories for those patients with smoking-related COPD attending pulmonary clinics that have reached their 60s, and it is possible that the proportion of individuals belonging to each trajectory will vary over time as obesity becomes more prevalent over the last 30 years. Finally, we must be cautious about establishing causal inference between the type of trajectory and final phenotype. Nevertheless, we used the group-based trajectory modelling method, which is a hypothesis-free tool aimed to assign a membership to those participants with similar trajectories, without a priori inclusion of their clinical characteristics [41]. Once participants were assigned to a trajectory by the method, we compared their clinical characteristics. This sequence is particularly helpful to mitigate selection bias and provides strong evidence for the distinction between trajectories and specific phenotypes. There is no doubt that the ideal methodology to address our hypothesis is to conduct a population-based study that includes subjects 18 years old (or younger) at risk of developing COPD with at least 40 years of follow-up. This is unlikely to be conducted. Unfortunately, most COPD epidemiological studies have a relatively short observation period [42, 43], and there may not be detailed measurements over time to define the specific phenotypes in the cohorts used to establish lung function trajectories [2].

Our findings highlight the need to study the determinants for distinct COPD phenotypes and opens the possibility of establishing trajectories in the different dimensions that are known to exist in COPD. It confirms that we should look beyond the $\mathrm{FEV}_{1}$ rate of decline to better understand the biology behind different phenotypes despite a single common exposure (tobacco smoking). Longitudinal follow-up studies should be carried out in cohorts established at an early age to determine anthropometric lung function beyond spirometry, imaging and biomarkers, aimed at deepening our understanding of the biology of the disease in its early stages. More specifically our findings suggest that adipose tissue is not just a fat deposit and may play a modulating role in the genesis of COPD.

In conclusion, our study provides evidence that individuals who develop COPD have distinct somatotype trajectories throughout adulthood leading to specific phenotypes. Subjects with the MOLT phenotype are programmed from an early age to maintain a lean trajectory through adult life. This particular subgroup of smokers deserves special attention as they are likely to become those patients with the most severe form of COPD.

Author contributions: The above listed authors attest that they made substantial contributions to the conception and design, acquisition of data, or analysis and interpretation of data; drafting the article or revising it critically for important intellectual content; and final approval of the version to be submitted for revision. All authors had full access to all the data in the study and accept responsibility for the submission of this work.

Conflict of interest: M.J. Divo has nothing to disclose. M. Marin Oto has nothing to disclose. C. Casanova Macario reports, in the last 3 years, to have received lectures and/or scientific advice from Laboratorios Bial, Boehringer Ingelheim, Gebropharma, GSK, Esteve, Menarini, Novartis and Rovi. C. Cabrera Lopez has nothing to disclose. J.P. de-Torres has nothing to disclose. J.M. Marin Trigo has nothing to disclose. C.P. Hersh reports grants from National Institutes of Health during the conduct of the study; and grants from Boehringer Ingelheim and Novartis, and personal fees from 23 and Me, outside the submitted work. A. Ezponda Casajús has nothing to disclose. C. Maguire has nothing to disclose. V.M. Pinto-Plata has nothing to disclose. F. Polverino has nothing to disclose. J.C. Ross reports grants from NIH during the conduct of the study. D. DeMeo has nothing to disclose. G. Bastarrika reports personal fees from General Electric, nonfinancial support from Siemens and grants from Guerbet, outside the submitted work. E.K. Silverman reports grants from NIH during the conduct of the study, and grants and travel support from GlaxoSmithKline outside the submitted work. B.R. Celli has nothing to disclose. 
Support statement: The COPDGene cohort (NCT00608764) is funded by National Institutes of Health (NIH) grants U01HL089856 and U01HL089897 and is also supported by the COPD Foundation through contributions made to an Industry Advisory Committee comprised of AstraZeneca, Boehringer Ingelheim, GlaxoSmithKline, Novartis, and Sunovion. Funding information for this article has been deposited with the Crossref Funder Registry.

\section{References}

$1 \quad$ Martinez FD. Early-life origins of chronic obstructive pulmonary disease. N Engl J Med 2016; 375: 871-878.

2 Lange P, Celli B, Agusti A, et al. Lung-function trajectories leading to chronic obstructive pulmonary disease. N Engl J Med 2015; 373: 111-122.

3 Dornhorst AC. Respiratory insufficiency. Lancet 1955; 268: 1185-1187.

4 Burgel PR, Paillasseur JL, Caillaud D, et al. Clinical COPD phenotypes: a novel approach using principal component and cluster analyses. Eur Respir J 2010; 36: 531-539.

5 Burgel P-R, Paillasseur J-L, Janssens W, et al. A simple algorithm for the identification of clinical COPD phenotypes. Eur Respir J 2017; 50: 1701034

6 Castaldi PJ, Dy J, Ross J, et al. Cluster analysis in the COPDGene study identifies subtypes of smokers with distinct patterns of airway disease and emphysema. Thorax 2014; 69: 415-422.

7 Garcia-Aymerich J, Gómez FP, Benet M, et al. Identification and prospective validation of clinically relevant chronic obstructive pulmonary disease (COPD) subtypes. Thorax 2011; 66: 430-437.

8 Rennard SI, Locantore N, Delafont B, et al. Identification of five chronic obstructive pulmonary disease subgroups with different prognoses in the ECLIPSE cohort using cluster analysis. Ann Am Thorac Soc 2015; 12: 303-312.

9 Castaldi PJ, Benet M, Petersen H, et al. Do COPD subtypes really exist? COPD heterogeneity and clustering in 10 independent cohorts. Thorax 2017; 72: 998-1006.

10 Celli BR, Locantore N, Tal-Singer R, et al. Emphysema and extrapulmonary tissue loss in COPD: a multi-organ loss of tissue phenotype. Eur Respir J 2018; 51: 1702146.

11 Agustí A, Barberà JA, Wouters EFM, et al. Lungs, bone marrow, and adipose tissue. A network approach to the pathobiology of chronic obstructive pulmonary disease. Am J Respir Crit Care Med 2013; 188: 1396-1406.

12 Song $\mathrm{M}, \mathrm{Hu} \mathrm{FB}, \mathrm{Wu}$, et al. Trajectory of body shape in early and middle life and all cause and cause specific mortality: results from two prospective US cohort studies. BMJ 2016; 353: i2195-i2110.

13 Zheng Y, Song M, Manson JE, et al. Group-based trajectory of body shape from ages 5 to 55 years and cardiometabolic disease risk in 2 US cohorts. Am J Epidemiol 2017; 186: 1246-1255.

14 Song M, Willett WC, Hu FB, et al. Trajectory of body shape across the lifespan and cancer risk. Int J Cancer 2016; 138: $2383-2395$

15 Regan EA, Hokanson JE, Murphy JR, et al. Genetic epidemiology of COPD (COPDGene) study design. COPD 2010; 7: 32-43.

16 Celli BR, Cote CG, Marin JM, et al. The body-mass index, airflow obstruction, dyspnea, and exercise capacity index in chronic obstructive pulmonary disease. N Engl J Med 2004; 350: 1005-1012.

17 Stunkard AJ, Sorenson T, Schulsinger F, et al. Use of the Danish Adoption Register for the study of obesity and thinness. In: Kety SS, Rowland LP, Sidman RL, eds. The Genetics of Neurological and Psychiatric Disorders. New York, NY, Raven Press, 1983; pp. 115-120.

18 Must A, Willett WC, Dietz WH. Remote recall of childhood height, weight, and body build by elderly subjects. Am J Epidemiol 1993; 138: 56-64.

19 Keshtkar AA, Semnani S, Pourshams A, et al. Pictogram use was validated for estimating individual's body mass index. J Clin Epidemiol 2010; 63: 655-659.

20 van Sloten TT, Boutouyrie P, Lisan Q, et al. Body silhouette trajectories across the lifespan and vascular aging. Hypertension 2018; 72: 1095-1102.

21 Laszlo G. Standardisation of lung function testing: helpful guidance from the ATS/ERS Task Force. Thorax 2006 61: 744-746.

22 ATS Committee on Proficiency Standards for Clinical Pulmonary Function Laboratories. ATS statement guidelines for the six-minute walk test. Am J Respir Crit Care Med 2002; 166: 111-117.

23 Bankier AA, Gevenois PA, Madani A. CT Quantification of pulmonary emphysema: assessment of lung structure and function. Crit Rev Computed Tomography 2002; 43: 397-415.

24 Bergin C, Müller N, Nichols DM, et al. The diagnosis of emphysema. A computed tomographic-pathologic correlation. Am Rev Respir Dis 1986; 133: 541-546.

25 Higgins MW, Keller JB, Becker M, et al. An index of risk for obstructive airways disease. Am Rev Respir Dis 1982; 125: 144-151.

26 Lee JM, Pilli S, Gebremariam A, et al. Getting heavier, younger: trajectories of obesity over the life course. Int J Obes 2010; 34: 614-623.

27 Harik-Khan RI, Fleg JL, Wise RA. Body mass index and the risk of COPD. Chest 2002; 121: 370-376.

28 Schols AMWJ, Broekhuizen R, Weling-Scheepers CA, et al. Body composition and mortality in chronic obstructive pulmonary disease. Am J Clin Nutr 2005; 82: 53-59.

29 Schols AM, Slangen J, Volovics L, et al. Weight loss is a reversible factor in the prognosis of chronic obstructive pulmonary disease. Am J Respir Crit Care Med 1998; 157: 1791-1797.

30 Jee SH, Sull JW, Park J, et al. Body-mass index and mortality in Korean men and women. N Engl J Med 2006; 355: 779-787.

31 Divo MJ, Cabrera C, Casanova C, et al. Comorbidity distribution, clinical expression and survival in COPD patients with different body mass index. Chronic Obstr Pulm Dis 2014; 1: 229-238.

32 Celli BR. COPD: time to improve its taxonomy? ERJ Open Res 2018; 4: 1-8.

33 Agustí A, Celli B, Faner R. What does endotyping mean for treatment in chronic obstructive pulmonary disease? Lancet 2017; 390: 980-987.

34 Sood A. Obesity, adipokines, and lung disease. J Appl Physiol 2010; 108: 744-753.

35 Bonfield TL, Caplan AI. Adult mesenchymal stem cells: an innovative therapeutic for lung diseases. Discov Med 2010; 9: 337-345. 
Frayling TM, Timpson NJ, Weedon MN, et al. A common variant in the FTO gene is associated with body mass index and predisposes to childhood and adult obesity. Science 2007; 316: 889-894.

37 Hinney A, Nguyen TT, Scherag A, et al. Genome wide association (GWA) study for early onset extreme obesity supports the role of fat mass and obesity associated gene (FTO) variants. PLoS One 2007; 2: e1361.

38 Song M, Zheng Y, Qi L, et al. Associations between genetic variants associated with body mass index and trajectories of body fatness across the life course: a longitudinal analysis. Int J Epidemiol 2018; 47: 506-515.

39 Wan ES, Cho MH, Boutaoui N, et al. Genome-wide association analysis of body mass in chronic obstructive pulmonary disease. Am J Respir Cell Mol Biol 2011; 45: 304-310.

40 Feinstein AR. Directionality and scientific inference. J Clin Epidemiol 1989; 42: 829-833.

41 Nagin DS, Jones BL, Passos VL, et al. Group-based multi-trajectory modeling. Stat Methods Med Res 2018; 27: 2015-2023.

42 Rutten EPA, Calverley PMA, Casaburi R, et al. Changes in body composition in patients with chronic obstructive pulmonary disease: do they influence patient-related outcomes? Ann Nutr Metab 2013; 63: 239-247.

43 Rutten EPA, Spruit MA, McDonald M-LN, et al. Continuous fat-free mass decline in COPD: fact or fiction? Eur Respir J 2015; 46: 1496-1498. 